\title{
The Value Compressive Strength and Split Tensile Strength on Concrete Mixture With Expanded Polystyrene Coated by Surfactant Span 80 as a Partial Substitution of Fine Aggregate
}

\author{
Irpan Hidayat $^{1}$, Alice Siauwantara ${ }^{1}$ \\ ${ }^{1}$ Bina Nusantara University, KH Syahdan 9, West Jakarta 11480, Indonesia
}

\begin{abstract}
The value of the density normal concrete which ranges between $2200-2400 \mathrm{~kg} / \mathrm{m}^{3}$. Therefore the use of Expanded Polystyrene (EPS) as a subitute to fine aggregate can reduce the density of concrete. The purpose this research is to reduce the density of normal concrete but increase compressive strength of EPS concrete, with use surfactant as coating for the EPS. Variables of substitution percentage of EPS and EPS coated by surfactant are 5\%,10\%,15\%,20\%,25\%. Method of concrete mix design based on SNI 03-2834-2000 "Tata Cara Pembuatan Rencana Campuran Beton Normal (Provisions for Proportioning Normal Concrete Mixture)". The result of testing, every increase percentage of EPS substitution will decrease the compressive strength around 1,74 $M P a$ and decrease density $34,03 \mathrm{~kg} / \mathrm{m}^{3}$. Using Surfactant as coating of EPS, compressive strength increase from the EPS's compressive strength. Average of increasing compressive strength 0,19 MPa and increase the density $20,03 \mathrm{~kg} / \mathrm{m}^{3}$, average decrease of the tensile split strength EPS coated surfaktan is 0,84 MPa.
\end{abstract}

\section{Introduction}

Along with technological developments in the field of construction, using concrete materials are commonly used as a material that supports the building structure . Theoretically, the concrete material is able to resist high compressive strength, but not good enough to resist tensile strength. The value of the density normal concrete which ranges between $2200-2400 \mathrm{~kg} / \mathrm{m}^{3}$, this will affect the total weight of the building structure. The decreasing density of the concrete will reduce the weight of the building.

To reduce the density of normal concrete, concrete toward research that has less weight is being carried out at several universities or in the concrete mix design company. One way to reduce the density of normal concrete by reducing the fine aggregate, material to be added Expanded Polystyrene (EPS) in the concrete mix. So in this way is expected that the resulting concrete material has a light density. Park and Chisholm use polystyrene as fine aggregate and has a less specific gravity ranges from 520 to $1040 \mathrm{~kg} / \mathrm{m} 3$ has a very low compressive strength, which is in the range $0.7 \mathrm{MPa}$ to 6.7 MPa

The reduced density of the concrete mixture will cause a decrease in the compressive strength of concrete. In this study will be seen how much the decrease of weight in the normal concrete mix if the substitution EPS is done on fine aggregate. To increase the compressive strength in concrete mix using EPS material substitution, we will perform an EPS coating material with chemical additives are surfactants span 80 . So expect the presence of EPS coating with surfactant span 80 can increase the value of the concrete compressive strength.

\section{Research Significance}

The purpose of this study was to analyze the influence of a mixture of normal concrete by reducing the fine aggregate material for later substituted with EPS, and EPS coated by surfactant span 80 . In the concrete mixtures, will be reviewed against the weight of concrete, concrete compressive strength and split tensile strength.

The expected benefits are the results of this research can be developed to generate concrete composition of lighter material with the compressive strength of concrete is higher than the EPS concrete mix that is not coated with surfactant.

The following are the limitations issue in this research is the cement used was Portland cement type 1, the design methods used to using SNI 03-2834-2000 with plan compressive strength of $25 \mathrm{MPa}$, the concrete samples will be formed with a cylindrical mold with $30 \mathrm{~cm}$ high and $15 \mathrm{~cm}$ in diameter, the size of EPS used 1-2 $\mathrm{mm}$ diameter, material substitution EPS composition was 5\%, $10 \%, 15 \%, 20 \%, 25 \%$, the surfactant used is span 80 .

\section{Test Program and Measurements}

Testing to be performed in this study is testing the compressive strength and split tensile strength. Based on the results, the study will be analyzed value of density, compressive strength and split tensile strength for normal concrete mixes, concrete mixes with EPS, and concrete mixture that surfactant span 80 as coating of EPS. The following concrete testing to be performed : 


\subsection{Compressive Strength}

Compressive strength is the ability of concrete to resist compressive strength with a certain weight on the concrete surface area. The tools are used for testing the compressive strength test is a compression test. Compressive strength formula are used by SNI 03-19742000:

Compressive strength concrete $: \frac{\mathrm{P}}{\mathrm{A}}$

Description:

$\mathrm{P}=$ maximum load $(\mathrm{kg})$

A $=$ cross-sectional area of the test sample $\left(\mathrm{cm}^{2}\right)$

The steps compressive strength testing that prepare specimens already in curing many as 3 samples, consider and record the weight of the sample, place the sample in the testing test, providing a constant load on the concrete until the concrete failure and noted the ability to resist maximum concrete compressive strength, tests performed at 28 days.

\subsection{Split Tensile Strength}

Tensile strength of concrete is the ability to resist the concrete tensile strength. Tensile test equipment that is used is the Universal Testing Machine. Provisions of tensile strength testing of concrete by SNI 03-2491-2002. Tensile strength of concrete formula:

$$
f_{c t}: \frac{2 P}{L D}
$$

Description:

$\mathrm{f}_{\mathrm{ct}}=$ split tensile strength $(\mathrm{MPa})$

$\mathrm{P}=$ maximum test load $(\mathrm{N})$

$\mathrm{L}=$ length of the sample $(\mathrm{mm})$

$\mathrm{D}=$ diameter of the sample $(\mathrm{mm})$

The steps tensile strength testing of concrete that prepare 3 pieces of concrete sample at concrete age of 28 days and perform tensile testing of concrete by using Universal Testing Machine tools.

\section{Test Results and Analysis}

Concrete mixture proportion is designed under the provisions of SNI 03-2834-2002, Tata Cara Pembuatan Rencana Campuran Beton Normal (Provisions for Proportioning Normal Concrete Mixture). The material proportions required per $\mathrm{m} 3$ for each EPS percentage are shown in the Table 1.

Table 1 Proportion Mixed Concrete

\begin{tabular}{|c|c|c|c|c|c|c|c|}
\hline \multirow{3}{*}{ Variabel } & \multirow{3}{*}{ Volume } & \multicolumn{6}{|c|}{ Proportion Mixed Concrete } \\
\hline & & \multirow{2}{*}{$\begin{array}{c}\text { Coarse } \\
\text { Aggregate } \\
\text { (kg) }\end{array}$} & \multirow{2}{*}{$\begin{array}{c}\text { Fine } \\
\text { Aggregate } \\
\text { (kg) }\end{array}$} & \multirow{2}{*}{$\begin{array}{c}\text { Cement } \\
(\mathrm{kg})\end{array}$} & \multirow{2}{*}{$\begin{array}{l}\text { Water } \\
\text { (L) }\end{array}$} & \multicolumn{2}{|c|}{$\begin{array}{c}\text { Subtitution } \\
\text { Fine Aggregate }\end{array}$} \\
\hline & & & & & & EPS (gr) & $\begin{array}{c}\text { Sand } \\
(\mathrm{Kg})\end{array}$ \\
\hline Normal & $1 \mathrm{~m}^{3}$ & 1164,92 & 505,29 & 355,77 & 159,01 & - & 505,29 \\
\hline EPS 5\% & $1 \mathrm{~m}^{3}$ & 1140,65 & 589,06 & 355,77 & 99,53 & 358,02 & $\overline{559,61}$ \\
\hline EPS $10 \%$ & $1 \mathrm{~m}^{3}$ & 1141,55 & 549,55 & 355,77 & 138,13 & 668,01 & 494,59 \\
\hline EPS $15 \%$ & $1 \mathrm{~m}^{3}$ & 1140,65 & 589,06 & 355,77 & 99,53 & 1074,05 & 500,70 \\
\hline EPS $20 \%$ & $1 \mathrm{~m}^{3}$ & 1140,65 & 537,27 & 355,77 & 151,31 & 1306,17 & 429,82 \\
\hline EPS $25 \%$ & $1 \mathrm{~m}^{3}$ & 1140,65 & 589,06 & 355,77 & 99,53 & 1790,09 & 441,79 \\
\hline EPS surfactant $5 \%$ & $1 \mathrm{~m}^{3}$ & 1151,99 & 504,10 & 355,77 & 173,14 & 593,39 & 478,90 \\
\hline EPS surfactant $10 \%$ & $1 \mathrm{~m}^{3}$ & 1151,99 & 515,32 & 355,77 & 161,92 & 1213,18 & 412,25 \\
\hline EPS surfactant $15 \%$ & $1 \mathrm{~m}^{3}$ & 1121,36 & 515,73 & 355,77 & 192,15 & 1821,21 & 438,37 \\
\hline EPS surfactant $20 \%$ & $1 \mathrm{~m}^{3}$ & 1121,36 & 515,73 & 355,77 & 192,15 & 2428,28 & 412,58 \\
\hline EPS surfactant $25 \%$ & $1 \mathrm{~m}^{3}$ & 1117,96 & 529,79 & 355,77 & 181,48 & 3118,15 & 397,35 \\
\hline
\end{tabular}

From the results of laboratory tests performed on the data obtained by the compressive strength and split tensile strength of concrete at 28 days. Tests carried out for 3 samples for each variable. Here are the results of testing the compressive strength and tensile strength of normal concrete, with EPS concrete mixture, and mix concrete with EPS coated by surfactant span 80 .

\subsection{Compressive Strength Test Results}

Tables 2 and 3 are the results of testing the compressive strength of concrete mix for EPS and EPS were coated by surfactant. 
Table 2 Compressive Strength Concrete EPS at 28 days

\begin{tabular}{|c|c|c|c|c|c|c|c|c|c|}
\hline \multirow[t]{2}{*}{ No } & \multirow[t]{2}{*}{ Variabel } & $\begin{array}{c}\text { Volume } \\
\text { Sample } \\
\left(\mathrm{m}^{3}\right)\end{array}$ & $\begin{array}{c}\text { Area } \\
\text { Sampel } \\
\left(\mathbf{m m}^{2}\right)\end{array}$ & \multirow[t]{2}{*}{$\begin{array}{c}\text { Weight } \\
\text { (kg) }\end{array}$} & $\begin{array}{c}\text { Experi } \\
\text { ment } \\
\text { Test } \\
\text { Results } \\
\text { (kN) }\end{array}$ & $\begin{array}{l}\text { Compressive } \\
\text { Strength at } \\
28 \text { days } \\
\text { (MPa) }\end{array}$ & $\begin{array}{c}\text { Compressive } \\
\text { Strength } \\
\text { Average } \\
\text { (MPa) }\end{array}$ & $\begin{array}{c}\text { Weight } \\
\text { Average } \\
\text { (kg) }\end{array}$ & $\begin{array}{l}\text { Density } \\
\left(\mathrm{kg} / \mathrm{m}^{3}\right)\end{array}$ \\
\hline & & $\begin{array}{c}v= \\
\pi r^{2} t\end{array}$ & $A=\pi r^{2}$ & & (P) & $\begin{array}{c}\mathbf{f}^{\prime} \mathbf{c}= \\
(\mathbf{P x} 1000) / \mathrm{A}\end{array}$ & f'cr & br & $\mathbf{b r} / \mathbf{v}$ \\
\hline \multirow{3}{*}{1} & \multirow{3}{*}{ EPS $0 \%$} & \multirow{3}{*}{0,0053} & \multirow{3}{*}{17662,5} & 11,43 & 420 & 23,78 & \multirow{3}{*}{24,53} & \multirow{3}{*}{11,58} & \multirow{3}{*}{2184,79} \\
\hline & & & & 11,675 & 450 & 25,48 & & & \\
\hline & & & & 11,625 & 430 & 24,35 & & & \\
\hline \multirow{3}{*}{2} & \multirow{3}{*}{ EPS 5\% } & \multirow{3}{*}{0,0053} & \multirow{3}{*}{17662,5} & 11,438 & 350 & 19,82 & \multirow{3}{*}{19,63} & \multirow{3}{*}{11,2} & \multirow{3}{*}{2113,14} \\
\hline & & & & 11,129 & 350 & 19,82 & & & \\
\hline & & & & 11,024 & 340 & 19,25 & & & \\
\hline \multirow{3}{*}{3} & \multirow{3}{*}{ EPS $10 \%$} & \multirow{3}{*}{0,0053} & \multirow{3}{*}{17662,5} & 11,05 & 330 & 18,68 & \multirow{3}{*}{18,12} & \multirow{3}{*}{11,07} & \multirow{3}{*}{2089,11} \\
\hline & & & & 11,02 & 330 & 18,68 & & & \\
\hline & & & & 11,139 & 300 & 16,99 & & & \\
\hline \multirow{3}{*}{4} & \multirow{3}{*}{ EPS $15 \%$} & \multirow{3}{*}{0,0053} & \multirow{3}{*}{17662,5} & 10,796 & 310 & 17,55 & \multirow{3}{*}{16,99} & \multirow{3}{*}{11} & \multirow{3}{*}{2076,34} \\
\hline & & & & 11,107 & 290 & 16,42 & & & \\
\hline & & & & 11,103 & 300 & 16,99 & & & \\
\hline \multirow{3}{*}{5} & \multirow{3}{*}{ EPS $20 \%$} & \multirow{3}{*}{0,0053} & & 10,879 & 280 & 15,85 & & & \\
\hline & & & 17662,5 & 10,867 & 290 & 16,42 & 16,23 & 10,88 & 2053,06 \\
\hline & & & & 10,89 & 290 & 16,42 & & & \\
\hline & & & & 10,565 & 320 & 18,12 & & & \\
\hline 6 & EPS $25 \%$ & 0,0053 & 17662,5 & 10,58 & 290 & 16,42 & 15,85 & 10,68 & 2014,63 \\
\hline & & & & 10,88 & 230 & 13,02 & & & \\
\hline
\end{tabular}

Table 3 Compressive Strength Concrete EPS Coated by Surfactant at 28 Days

\begin{tabular}{|c|c|c|c|c|c|c|c|c|c|}
\hline \multirow[t]{2}{*}{ No } & \multirow[t]{2}{*}{ Variabel } & $\begin{array}{c}\text { Volume } \\
\text { Sample } \\
\left(\mathrm{m}^{3}\right)\end{array}$ & $\begin{array}{c}\text { Area } \\
\text { Sampel } \\
\left(\mathbf{m m}^{2}\right)\end{array}$ & \multirow[t]{2}{*}{$\begin{array}{c}\text { Weight } \\
\text { (kg) }\end{array}$} & $\begin{array}{l}\text { Experi } \\
\text { ment } \\
\text { Test } \\
\text { Results } \\
(\mathbf{k N})\end{array}$ & $\begin{array}{l}\text { Compressive } \\
\text { Strength at } \\
28 \text { days } \\
\text { (MPa) }\end{array}$ & $\begin{array}{l}\text { Compressive } \\
\text { Strength } \\
\text { Average } \\
\text { (MPa) }\end{array}$ & $\begin{array}{c}\text { Weight } \\
\text { Average } \\
\text { (kg) }\end{array}$ & $\begin{array}{l}\text { Density } \\
\left(\mathrm{kg} / \mathrm{m}^{3}\right)\end{array}$ \\
\hline & & $\begin{array}{c}v= \\
\pi r^{2} t\end{array}$ & $A=\pi r^{2}$ & & (P) & $\begin{array}{c}f^{\prime} c= \\
(P \times 1000) / A\end{array}$ & fer & br & $\mathbf{b r} / \mathbf{v}$ \\
\hline \multirow{3}{*}{1} & \multirow{3}{*}{ EPSS $5 \%$} & \multirow{3}{*}{0,0053} & \multirow{3}{*}{17662,5} & 11,112 & 350 & 19,82 & \multirow{3}{*}{19,48} & \multirow{3}{*}{11,34} & \multirow{3}{*}{2140,65} \\
\hline & & & & 11,43 & 350 & 19,82 & & & \\
\hline & & & & 11,458 & 350 & 19,82 & & & \\
\hline \multirow{3}{*}{2} & \multirow{3}{*}{ EPSS 10\% } & \multirow{3}{*}{0,0053} & \multirow{3}{*}{17662,5} & 11,08 & 320 & 18,12 & \multirow{3}{*}{18} & \multirow{3}{*}{11,18} & \multirow{3}{*}{2109,32} \\
\hline & & & & 11,311 & 320 & 18,12 & & & \\
\hline & & & & 11,245 & 330 & 18,68 & & & \\
\hline \multirow{3}{*}{3} & \multirow{3}{*}{ EPSS 15\% } & \multirow{3}{*}{0,0053} & \multirow{3}{*}{17662,5} & 11,197 & 310 & 17,55 & \multirow{3}{*}{17,1} & \multirow{3}{*}{11,05} & \multirow{3}{*}{2085,54} \\
\hline & & & & 11,291 & 300 & 16,99 & & & \\
\hline & & & & 10,874 & 300 & 16,99 & & & \\
\hline \multirow{3}{*}{4} & \multirow{3}{*}{ EPSS 20\% } & \multirow{3}{*}{0,0053} & \multirow{3}{*}{17662,5} & 10,988 & 300 & 16,99 & \multirow{3}{*}{16,42} & \multirow{3}{*}{10,97} & \multirow{3}{*}{2070,94} \\
\hline & & & & 10,918 & 290 & 16,42 & & & \\
\hline & & & & 10,974 & 280 & 15,85 & & & \\
\hline \multirow{3}{*}{5} & \multirow{3}{*}{ EPSS $25 \%$} & \multirow{3}{*}{0,0053} & \multirow{3}{*}{17662,5} & 10,822 & 260 & 14,72 & \multirow{3}{*}{15,63} & & \\
\hline & & & & 10,900 & 310 & 17,55 & & 10,87 & 2051,84 \\
\hline & & & & 10,777 & 290 & 16,42 & & & \\
\hline
\end{tabular}




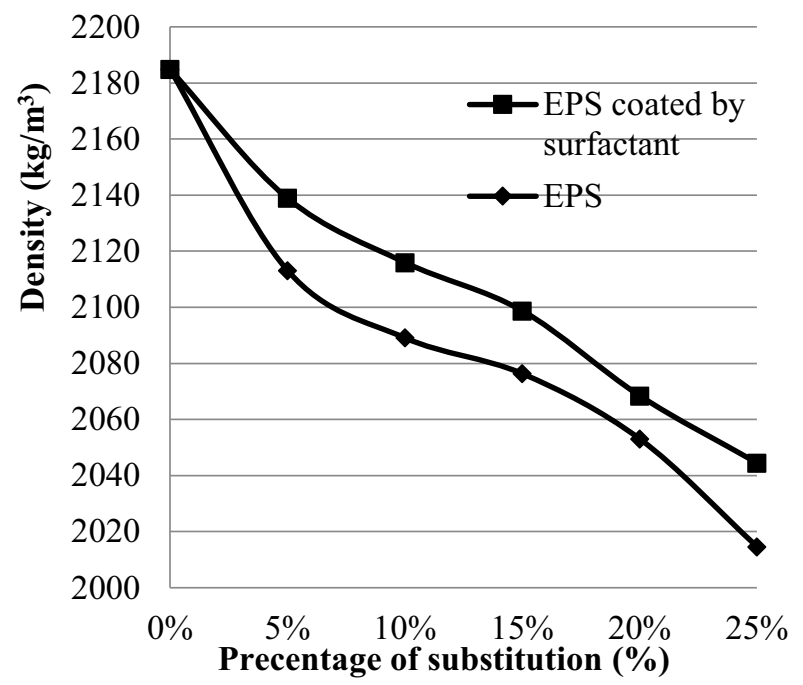

Figure 1 Percentage of EPS VS Density

According to the testing, Figure 1 can be shown that with the increasing percentage of substitution EPS will be reduced the density of the concrete. The density value will increase with the addition of a surfactant span 80 as coating of EPS in the concrete mix. Increasing the density of the concrete mixture EPS were coated surfactant also affect the compressive strength (figure 2). The compressive strength of concrete increases due to the influence of surfactant span 80 as coating of EPS, so the EPS granules become harder.

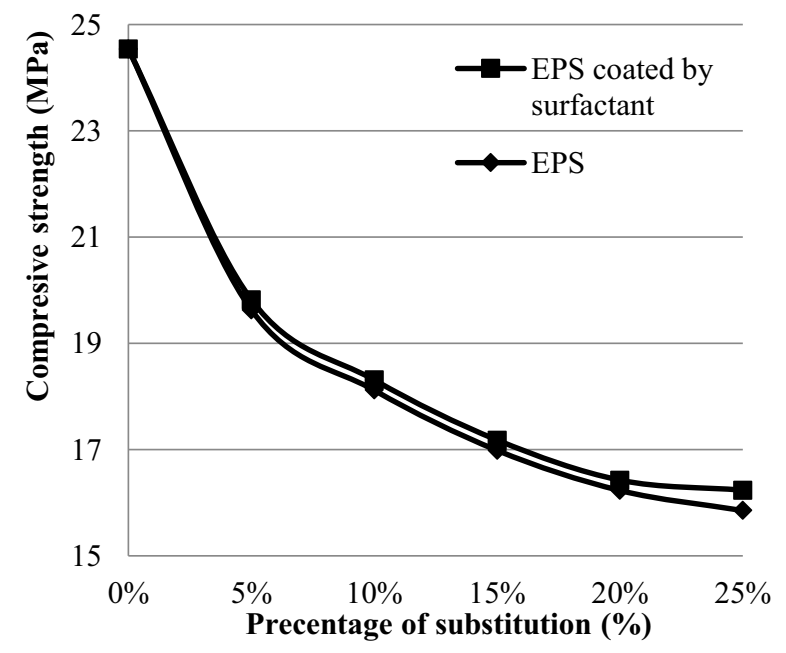

Figure 2 Percentage Compressive Strength vs EPS

Based on Figures 3 and 4, the increasing density of the concrete will increase the value of the compressive strength of concrete. This behavior occurs for concrete mixes with EPS and the EPS coated by surfactant span 80 .

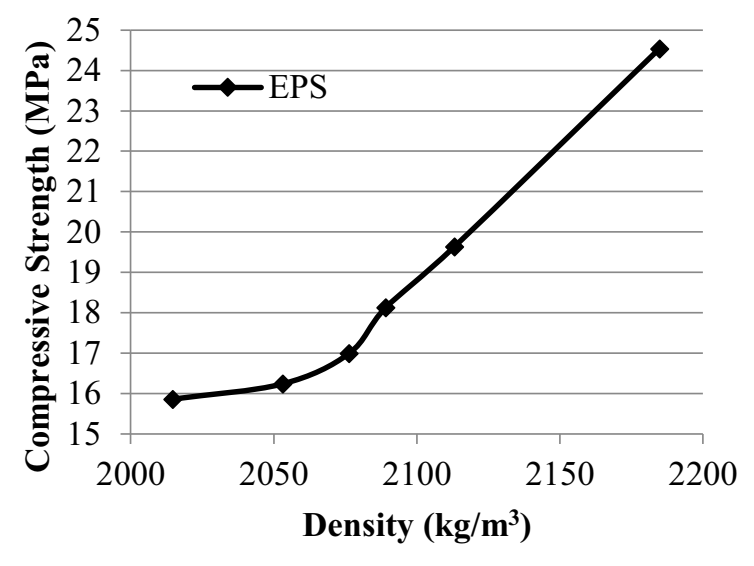

Figure 3 Density EPS vs. Concrete Compressive Strength

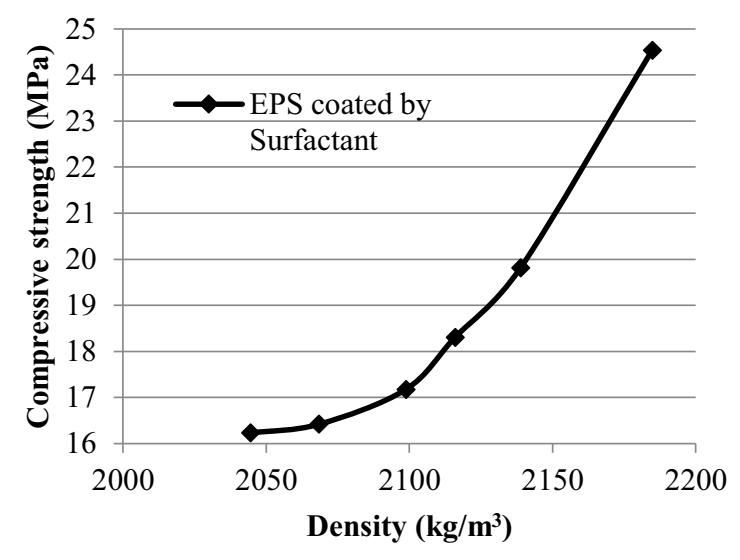

Figure 4 Density vs EPS coated by surfactant Concrete Compressive Strength

In Table 4, the difference or addition of compressive strength of concrete mix concrete EPS and EPS coated by surfactant having an average increase of concrete compressive strength of $0,19 \mathrm{MPa}$. From this testing it can be shown that the concrete mixture that EPS coated by surfactant can increase the compressive strength of concrete, but the value is not significant.

Table 4 Comparison of Concrete Compressive Strength EPS and EPS Coated by Surfactant

\begin{tabular}{|c|c|c|c|}
\hline$\%$ & EPS & $\begin{array}{c}\text { EPS } \\
\text { Surfactant } \\
\text { (MPa) }\end{array}$ & $\begin{array}{c}\text { Deviation } \\
\text { (MPa) }\end{array}$ \\
\hline $0 \%$ & 24,53 & 24,53 & 0,00 \\
\hline $5 \%$ & 19,63 & 19,82 & 0,19 \\
\hline $10 \%$ & 18,12 & 18,31 & 0,19 \\
\hline $15 \%$ & 16,99 & 17,17 & 0,19 \\
\hline $20 \%$ & 16,23 & 16,42 & 0,19 \\
\hline $25 \%$ & 15,85 & 16,23 & 0,38 \\
\hline \multicolumn{3}{|c|}{ Average } \\
\hline
\end{tabular}


Table 5 Comparison of Mixed Concrete Concrete Density EPS and EPS Coated by Surfactant

\begin{tabular}{|c|c|c|c|}
\hline $\begin{array}{c}\% \\
\text { Subtit } \\
\text { ution }\end{array}$ & $\begin{array}{c}\text { EPS } \\
\text { (MPa) }\end{array}$ & $\begin{array}{c}\text { EPS } \\
\text { surfactant } \\
\text { (MPa) }\end{array}$ & $\begin{array}{c}\text { Deviation } \\
\text { (MPa) }\end{array}$ \\
\hline $0 \%$ & 2184,79 & 2184,79 & 0,00 \\
\hline $5 \%$ & 2113,14 & 2138,869 & 25,73 \\
\hline $10 \%$ & 2089,11 & 2115,971 & 26,86 \\
\hline $15 \%$ & 2076,34 & 2098,734 & 22,40 \\
\hline $20 \%$ & 2053,06 & 2068,412 & 15,35 \\
\hline $25 \%$ & 2014,63 & 2044,444 & 29,82 \\
\hline \multicolumn{4}{|c|}{ Average } \\
\hline \multicolumn{3}{|c|}{} \\
\hline
\end{tabular}

Table 6 explain that every 5\% decrease in EPS for fine aggregate substitute in concrete mixtures obtained an average reduction of compressive strength of 1,74 $\mathrm{MPa}$ and an average density of $34,03 \mathrm{~kg} / \mathrm{m}^{3}$. Decreasing in the compressive strength of normal concrete mix concrete to the proportion of 5\% EPS amounted to 4,91 $\mathrm{MPa}$, the values differences by a margin decline EPS another substitution proportion $(10 \%, 15 \%, 20 \%, 25 \%)$.

Table 6 Difference of Decline Compressive Strength and Density EPS

\begin{tabular}{|c|c|c|c|c|}
\hline \multirow{2}{*}{ Variabel } & \multirow{2}{*}{$\mathbf{f} \mathbf{c}$} & \multirow{\mathbf{MPa}}{*}{} & $\begin{array}{c}\text { Density } \\
\left(\mathbf{K g} / \mathbf{m}^{\mathbf{3}}\right)\end{array}$ & $\begin{array}{c}\text { Decrease Deviation } \\
\text { EPS each 5\% }\end{array}$ \\
\hline & & $\begin{array}{c}\text { Compressive } \\
\text { Strength } \\
(\mathbf{M P a})\end{array}$ & $\begin{array}{c}\text { Density } \\
\left(\mathbf{K g} / \mathbf{m}^{3}\right)\end{array}$ \\
\hline $0 \%$ & 24,53 & 2184,79 & - & - \\
\hline $5 \%$ & 19,63 & 2113,14 & 4,91 & 71,65 \\
\hline $10 \%$ & 18,12 & 2089,11 & 1,51 & 24,03 \\
\hline $15 \%$ & 16,99 & 2076,34 & 1,13 & 12,77 \\
\hline $20 \%$ & 16,23 & 2053,06 & 0,75 & 23,28 \\
\hline $25 \%$ & 15,85 & 2014,63 & 0,38 & 38,44 \\
\hline \multicolumn{3}{|c|}{ Average } & 1,74 & 34,03 \\
\hline
\end{tabular}

Whereas decrease of the percentage substitution EPS 5 $\%$ were coated by surfactant in concrete mix, the average decrease in compressive strength of concrete of 1,66 $\mathrm{MPa}$ and a decrease in the average density of 28,07 $\mathrm{kg} / \mathrm{m}^{3}$. In Table 7 , it can be shown that any decrease in the density of the concrete it will also decrease the compressive strength of concrete.

Table 7 Difference of Decline Compressive Strength and Density EPS Coated by Surfactant

\begin{tabular}{|c|c|c|c|c|}
\hline \multirow[b]{2}{*}{ Variabel } & \multirow[b]{2}{*}{$\begin{array}{c}\text { f'c } \\
\text { (MPa) }\end{array}$} & \multirow[b]{2}{*}{$\begin{array}{l}\text { Density } \\
\left(\mathbf{K g} / \mathbf{m}^{3}\right)\end{array}$} & \multicolumn{2}{|c|}{$\begin{array}{l}\text { Decrease Deviation } \\
\text { EPS each 5\% }\end{array}$} \\
\hline & & & $\begin{array}{c}\text { f'c } \\
(\mathrm{MPa})\end{array}$ & $\begin{array}{l}\text { Density } \\
\left(\mathrm{Kg} / \mathrm{m}^{3}\right)\end{array}$ \\
\hline $0 \%$ & 24,53 & 2184,79 & & \\
\hline $5 \%$ & 19,82 & 2138,87 & 4,72 & 45,92 \\
\hline $10 \%$ & 18,31 & 2115,97 & 1,51 & 22,90 \\
\hline $15 \%$ & 17,17 & 2098,73 & 1,13 & 17,24 \\
\hline $20 \%$ & 16,42 & 2068,41 & 0,75 & 30,32 \\
\hline $25 \%$ & 16,23 & 2044,44 & 0,19 & 23,97 \\
\hline \multicolumn{3}{|c|}{ Average } & 1,66 & 28,07 \\
\hline
\end{tabular}

\subsection{Split Tensile Strength Test Results}

In addition to compressive strength test, the testing was also made of concrete split tensile strength test at the age of 28 days. The data will be obtained from tensile strength test of concrete mixtures with EPS and EPS were coated by surfactant as material substitution as fine aggregate.

The results of the split tensile strength testing for concrete at 28 days can be shown in Table 8. While in table 9, a split tensile strength and its density to mix concrete with EPS were coated by surfactant.

Table 8 Split Tensile Strength Concrete EPS at 28 days

\begin{tabular}{|c|c|c|c|c|c|c|c|c|c|}
\hline \multirow[t]{2}{*}{ Variabel } & $\begin{array}{c}\text { Height } \\
(\mathbf{m m})\end{array}$ & $\begin{array}{l}\text { Diameter } \\
\text { (mm) }\end{array}$ & $\begin{array}{c}\text { Volume } \\
\left(\mathbf{m}^{3}\right)\end{array}$ & \multirow[t]{2}{*}{$\begin{array}{c}\text { Weight } \\
\text { (kg) }\end{array}$} & $\begin{array}{c}\text { Experi } \\
\text { ment } \\
\text { Test } \\
\text { Results } \\
(\mathrm{kN})\end{array}$ & $\begin{array}{c}\text { Compressive } \\
\text { Strength at } 28 \\
\text { Days (MPa) }\end{array}$ & $\begin{array}{c}\text { Split } \\
\text { Tensile } \\
\text { Strength } \\
\text { Average } \\
\text { (MPa) }\end{array}$ & $\begin{array}{c}\text { Weight } \\
\text { Average } \\
\text { (kg) }\end{array}$ & $\begin{array}{l}\text { Density } \\
\left(\mathrm{kg} / \mathrm{m}^{3}\right)\end{array}$ \\
\hline & $\mathbf{L}$ & D & $\begin{array}{l}v \\
=\pi r^{2} t\end{array}$ & & $\mathbf{P}$ & $(2 \mathrm{P}) /(\mathrm{LD})$ & Fer & br & br/v \\
\hline \multirow{3}{*}{ Normal } & \multirow{3}{*}{150} & \multirow{3}{*}{300} & \multirow{3}{*}{0,0053} & 11,022 & 170 & 7,56 & \multirow{3}{*}{7,85} & \multirow{3}{*}{11,13} & \multirow{3}{*}{2099,74} \\
\hline & & & & 11,103 & 180 & 8,00 & & & \\
\hline & & & & 11,253 & 180 & 8,00 & & & \\
\hline \multirow{3}{*}{ EPS 5\% } & \multirow{3}{*}{150} & \multirow{3}{*}{300} & \multirow{3}{*}{0,0053} & 11,038 & 180 & 8,00 & \multirow{3}{*}{7,11} & \multirow{3}{*}{11,02} & \multirow{3}{*}{2079,11} \\
\hline & & & & 10,988 & 150 & 6,67 & & & \\
\hline & & & & 11,024 & 150 & 6,67 & & & \\
\hline \multirow{2}{*}{ EPS $10 \%$} & \multirow{2}{*}{150} & \multirow{2}{*}{300} & \multirow{2}{*}{0,0053} & 11,050 & 110 & 4,89 & \multirow{2}{*}{6,22} & \multirow{2}{*}{10,96} & \multirow{2}{*}{2068,98} \\
\hline & & & & 11,139 & 160 & 7,11 & & & \\
\hline
\end{tabular}




\begin{tabular}{|c|c|c|c|c|c|c|c|c|c|}
\hline & & & & 10,700 & 150 & 6,67 & & & \\
\hline \multirow{3}{*}{ EPS $15 \%$} & \multirow{3}{*}{150} & \multirow{3}{*}{300} & \multirow{3}{*}{0,0053} & 10,796 & 160 & 7,11 & \multirow{3}{*}{5,93} & \multirow{3}{*}{10,91} & \multirow{3}{*}{2059,61} \\
\hline & & & & 10,841 & 100 & 4,44 & & & \\
\hline & & & & 11,103 & 140 & 6,22 & & & \\
\hline \multirow{3}{*}{ EPS $20 \%$} & \multirow{3}{*}{150} & \multirow{3}{*}{300} & \multirow{3}{*}{0,0053} & 10,879 & 100 & 4,44 & \multirow{3}{*}{5,48} & \multirow{3}{*}{10,85} & \multirow{3}{*}{2046,77} \\
\hline & & & & 10,767 & 140 & 6,22 & & & \\
\hline & & & & 10,890 & 130 & 5,78 & & & \\
\hline \multirow{3}{*}{ EPS $25 \%$} & \multirow{3}{*}{150} & \multirow{3}{*}{300} & \multirow{3}{*}{0,0053} & 10,353 & 130 & 5,78 & \multirow{3}{*}{5,63} & \multirow{3}{*}{10,73} & \multirow{3}{*}{2025,51} \\
\hline & & & & 10,965 & 120 & 5,33 & & & \\
\hline & & & & 10,880 & 130 & 5,78 & & & \\
\hline
\end{tabular}

Table 9 Split Tensile Strength Concrete EPS Coated by Surfactant 28 days

\begin{tabular}{|c|c|c|c|c|c|c|c|c|c|}
\hline \multirow[t]{2}{*}{ Variabel } & $\begin{array}{c}\text { Height } \\
(\mathrm{mm})\end{array}$ & $\begin{array}{l}\text { Diameter } \\
(\mathrm{mm})\end{array}$ & $\begin{array}{c}\text { Volume } \\
\left(\mathbf{m}^{3}\right)\end{array}$ & \multirow[t]{2}{*}{$\begin{array}{c}\text { Weight } \\
\text { (kg) }\end{array}$} & $\begin{array}{c}\text { Experi } \\
\text { ment } \\
\text { Test } \\
\text { Results } \\
(\mathbf{k N}) \\
\end{array}$ & $\begin{array}{c}\text { Compressive } \\
\text { Strength at } 28 \\
\text { days MPa }\end{array}$ & $\begin{array}{c}\text { Split } \\
\text { Tensile } \\
\text { Strength } \\
\text { Average } \\
\text { (MPa) }\end{array}$ & $\begin{array}{c}\text { Weight } \\
\text { Average } \\
\text { (kg) }\end{array}$ & $\begin{array}{r}\text { Density } \\
\left(\mathrm{kg} / \mathrm{m}^{3}\right)\end{array}$ \\
\hline & $\mathbf{L}$ & D & $\begin{array}{l}v \\
=\pi r^{2} t\end{array}$ & & $\mathbf{P}$ & $(2 \mathrm{P}) /(\mathbf{L D})$ & Fer & br & $\mathrm{br} / \mathrm{v}$ \\
\hline \multirow{3}{*}{ EPSS $5 \%$} & \multirow{3}{*}{150} & \multirow{3}{*}{300} & \multirow{3}{*}{0,0053} & 11,021 & 140 & 6,22 & \multirow{3}{*}{5,93} & \multirow{3}{*}{11,08} & \multirow{3}{*}{2090,18} \\
\hline & & & & 11,012 & 140 & 6,22 & & & \\
\hline & & & & 11,193 & 120 & 5,33 & & & \\
\hline \multirow{3}{*}{ EPSS $10 \%$} & \multirow{3}{*}{150} & \multirow{3}{*}{300} & \multirow{3}{*}{0,0053} & 10,943 & 120 & 5,33 & \multirow{3}{*}{5,19} & \multirow{3}{*}{11,02} & \multirow{3}{*}{2080,11} \\
\hline & & & & 11,128 & 120 & 5,33 & & & \\
\hline & & & & 10,995 & 110 & 4,89 & & & \\
\hline \multirow{3}{*}{ EPSS $15 \%$} & \multirow{3}{*}{150} & \multirow{3}{*}{300} & \multirow{3}{*}{0,0053} & 10,997 & 100 & 4,44 & \multirow{3}{*}{4,89} & \multirow{3}{*}{10,99} & \multirow{3}{*}{2074,1} \\
\hline & & & & 11,091 & 110 & 4,89 & & & \\
\hline & & & & 10,882 & 120 & 5,33 & & & \\
\hline \multirow{3}{*}{ EPSS $20 \%$} & \multirow{3}{*}{150} & \multirow{3}{*}{300} & \multirow{3}{*}{0,0053} & 10,988 & 110 & 4,89 & \multirow{3}{*}{4,74} & \multirow{3}{*}{10,91} & \multirow{3}{*}{2059,01} \\
\hline & & & & 10,918 & 110 & 4,89 & & & \\
\hline & & & & 10,825 & 100 & 4,44 & & & \\
\hline \multirow{3}{*}{ EPSS $25 \%$} & \multirow{3}{*}{150} & \multirow{3}{*}{300} & \multirow{3}{*}{0,0053} & 10,882 & 110 & 4,89 & \multirow{3}{*}{4,59} & \multirow{3}{*}{10,85} & \\
\hline & & & & 10,779 & 100 & 4,44 & & & 2047,97 \\
\hline & & & & 10,894 & 100 & 4,44 & & & \\
\hline
\end{tabular}

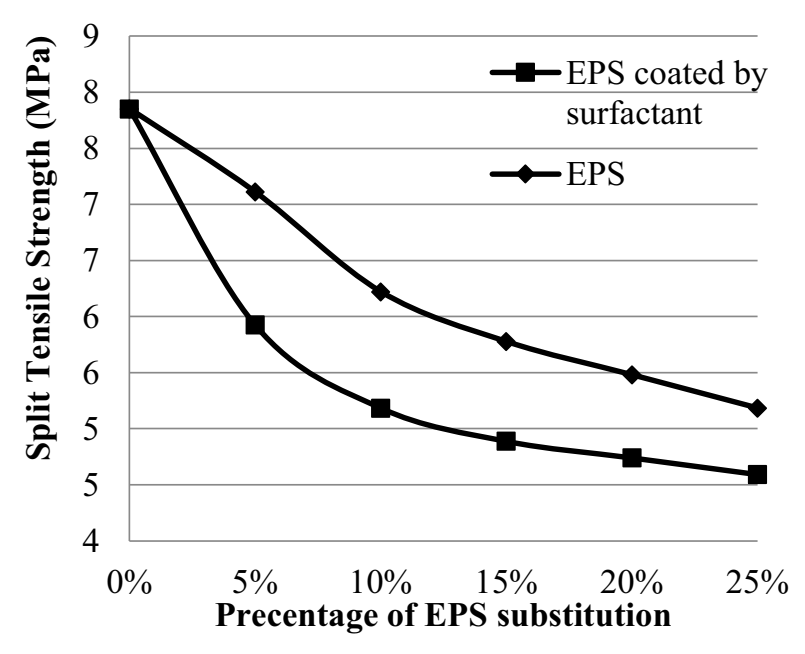

Figure 6 Percentage EPS to Split Tensile Strength
On Figure 6, can be shown a decline in the percentage of each additional substitution EPS and EPS were coated by surfactant as substitution the fine aggregate material. The addition of the surfactant as coating of EPS reduces the value of the tensile strength of the concrete of EPS.

\section{Conclusion}

Based on the results obtained the following conclusions:

- The substitution EPS of the fine aggregate in concrete mix then reduce the compressive strength, tensile strength and density of the normal concrete mix.

- The addition of surfactant 'span 80 as coating of EPS then increase compressive strength and density of the concrete mix, although not approaching the compressive strength and density of the normal concrete mix.

- $\quad$ The decrease of the average compressive strength when substitution EPS $5 \%$ of the fine aggregate with value $1,74 \mathrm{MPa}$, and the decrease of average density in the concrete mix is $34,03 \mathrm{~kg} / \mathrm{m}^{3}$. 
- The decrease of the average compressive strength when subtitution EPS $5 \%$ coated by surfactant of fine aggregate with value 1,66 $\mathrm{MPa}$, and a decrease in the average density of its concrete at $28,07 \mathrm{~kg} / \mathrm{m}^{3}$.

- The increase of the average compressive strength of the concrete mixes with EPS and EPS coated by surfactant is $0,19 \mathrm{MPa}$, and the increase in average density between EPS and EPS coated by surfactant in concrete mix is $20,03 \mathrm{~kg} / \mathrm{m}^{3}$.

- The maximum value of density and the compressive strength of concrete mix with EPS coated by surfactant is substituted EPS $5 \%$ of the fine aggregate. Where the value compressive strength and density of EPS coated by surfactant is $19,38 \mathrm{MPa}$ and $2140,565 \mathrm{~kg} / \mathrm{m}^{3}$.

- The addition of EPS coated by surfactant can reduces the split tensile strength values of 0.84 $\mathrm{MPa}$ for the split tensile strength of concrete mix with EPS.

- The maximum value of split tensile strength of concrete mixtures EPS when substitution EPS 5\% without surfactant, with a split tensile strength value of 7,11 $\mathrm{MPa}$. This value is still less than the split tensile strength of normal concrete mix $(7,85 \mathrm{MPa})$.

\section{References}

1. Badan Standardisasi Indonesia .SNI 03-2834-2000 : Tata Cara Pembuatan Rencana Campuran Beton Normal (Provisions for Proportioning Normal Concrete Mixture). Bandung : Badan Standardisasi Indonesia.( 2000).

2. Badan Standarisasi Indonesia. (2002). SNI 036815-2002 : Tata Cara Mengevaluasi Hasil Uji Kekuatan Beton (Provisions for Procedures for Evaluating Concrete Strength Test Results). Bandung : Badan Standardisasi Indonesia.

3. Badan Standardisasi Indonesia. (2004). SNI 152049-2004 : Semen Portland. Bandung : Badan Standardisasi Indonesia.

4. Park, S.G., and Chisholm, D.H. (1999). Polystyrene Aggregate Concrete. Study Report No. 85. Building Research Levy.

5. Giri, I. B. D., Sudarsana, I. K., dan Tutarani, N. M. Kuat Tekan Dan Modulus Elastisitas Beton Dengan Penambahan Styrofoam (Styrocon). Jurnal Ilmiah Teknik Sipil, 12(1), pp75-pp85. (2008).

6. Yusuf, R. (2011). Pengaruh Penggantian Pasir Dengan Expanded Polystyrene Terhadap Kuat Tekan Dan Berat Jenis Beton. Jakarta : Universitas Bina Nusantara.

7. Setyo, Dimas dan Puripangestuti, Risa (2007). Pengaruh Precoating Surfaktan Pada Expanded Polystyrene Sebagai Agregat Terhadap Struktur dan Properti Komposit Beton .Surabaya : ITS 